\title{
One-Step Preparation of Fuel-Containing Anisotropic Nanocapsules with Stimuli- Regulated Propulsion
}

Shuai Jiang, Anke Kaltbeitzel, Minghan Hu, Oksana Suraeva, Daniel Crespy,* and Katharina Landfester*

Cite This: ACS Nano 2020, 14, 498-508

Read Online

ACCESS | Lلll Metrics \& More | 回 Article Recommendations | st Supporting Information

ABSTRACT: One of the dreams of nanotechnology is to create tiny objects, nanobots, that are able to perform difficult tasks in dimensions and locations that are not directly accessible. One basic function of these nanobots is motility. Movements created by self-propelled micro- and nanovehicles are usually dependent on the production of propellants from catalytic reactions of fuels present in the environment. Developing self-powered nanovehicles with internally stored fuels that display motion regulated by external stimuli represents an intriguing and challenging alternative. Herein, a one-step preparation of fuelcontaining nanovehicles that feature a motion that can be regulated by external stimuli is reported. Nanovehicles are

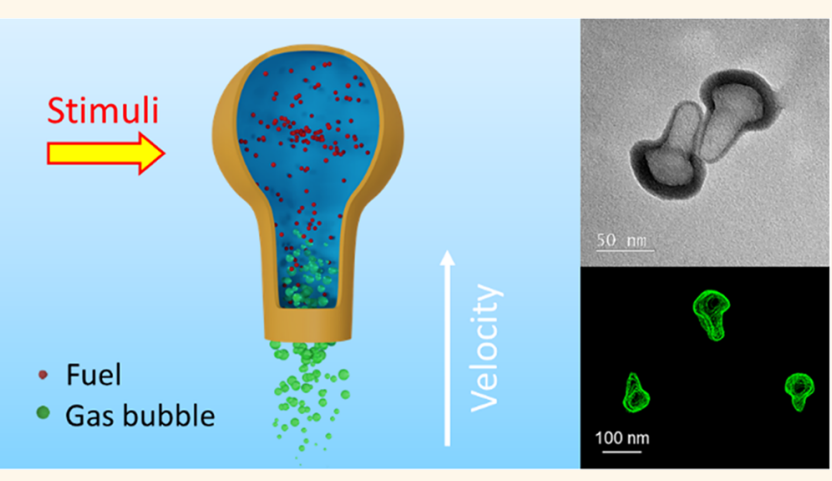
prepared via a sol-gel process confined at the oil/water interface of miniemulsions. The nanovehicles display shapes ranging from mushroom-like to truncated cones and a core-shell structure so that the silica shell acts as a hull for the nanovehicles while the core is used to store the fuel. Azo-based initiators are loaded in the nanovehicles, which are activated to release nitrogen gas upon increase of temperature or exposure to UV light. Enhanced diffusion of nanovehicles is achieved upon decomposition of the fuel.

KEYWORDS: asymmetric particles, core-shell nanoparticles, motility, nanocarriers, nanomotors

$\mathrm{B}$ iological molecular machines in living system harness energy molecules, such as from the hydrolysis of adenosine triphosphate (ATP), and translate them into mechanical activities. Rapid progress in nanotechnology enabled the artificial synthesis of micro- and nanovehicles for diverse applications, ranging from environmental remediation $^{1-3}$ to smart sensing ${ }^{4,5}$ and biomedical applications. ${ }^{6-9}$ In particular, nanovehicles with a size smaller than $100 \mathrm{~nm}$ show great potential for future molecular transport at the cellular level because they operate at length scales of protein motors. ${ }^{10,11}$ The self-propelling features of nanomotors can facilitate tissue penetration and crossing of cell membranes. ${ }^{12-14}$

Typically, external chemical energy from surrounding fuel molecules $^{11,13,15-20}$ or external fields, such as light, ${ }^{21,22}$ temperature gradients, ${ }^{23}$ and acoustic fields, ${ }^{24-28}$ is employed to provide the necessary driving force for self-propelled locomotion. This external dependence may however restrict the independence of the vehicles and make their actuation problematic once the external sources are inaccessible or variable in complex environments. ${ }^{29}$ Movement of catalytic vehicles, constituting an important class of nanovehicular systems, is generally fully dependent on the concentration of accessible fuels. The propulsive movement stops when the fuel is consumed completely. ${ }^{30} \mathrm{~A}$ built-in speed-regulation mechanism is therefore desirable. ${ }^{30}$ Moreover, many catalytic nanovehicles, with exceptions, ${ }^{31}$ are fabricated by top-down lithographic methods, which are time-intensive and difficult to scale up. The resulting nanovehicles are also usually not biocompatible. ${ }^{16}$ Thus, a bottom-up design of nanosized fuelcontaining vehicles is an attractive alternative but remains an intriguing challenge.

In this regard, we developed nanovehicles with a diameter of $\sim 100 \mathrm{~nm}$, a head-tail shape, core-shell structure, and selfcontained energy molecules in one structure that can be triggered in response to heat and light irradiation. There are three essential aspects for our design of the nanovehicles. (i) The core-shell structure allows the safe loading and protection

Received: August 13, 2019

Accepted: December 30, 2019

Published: December 30, 2019 

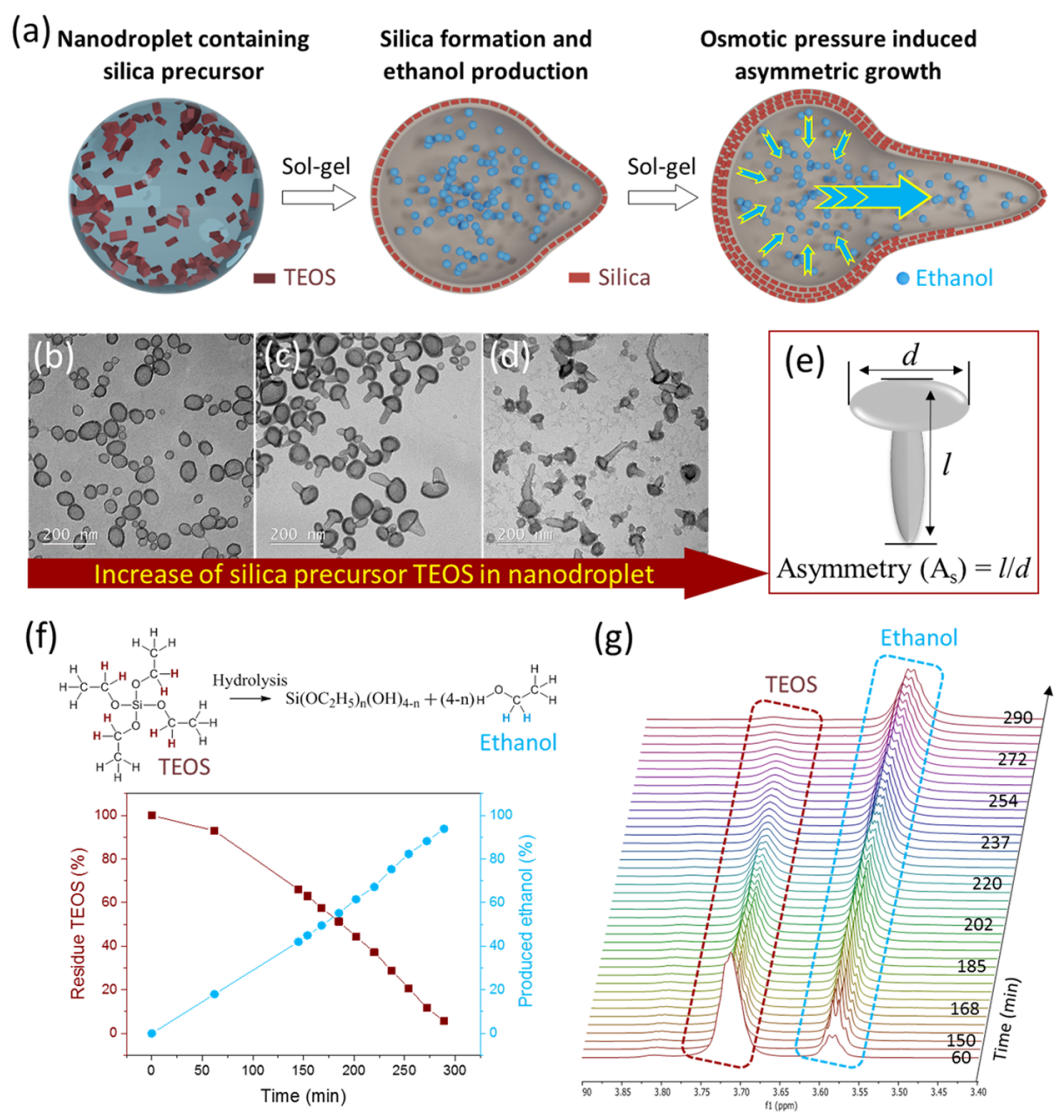

Figure 1. (a) Schematic illustration of the formation mechanism of asymmetric silica nanovehicles with core-shell structures. TEM micrographs of nanovehicles synthesized with different concentrations of TEOS in the miniemulsion droplets: (b) $63 \mathrm{wt} \%$, (c) $80 \mathrm{wt} \%$, and (d) $98 \mathrm{wt} \%$. (e) Representation of the asymmetric nanocapsules and definition of their relevant geometric parameters. (f,g) Kinetic study of TEOS hydrolysis and ethanol production during the formation of silica nanovehicles by ${ }^{1} \mathrm{H}$ NMR spectroscopy.

of fuel materials in the interior of the nanovehicles. (ii) Control of asymmetric shape at nanoscale is challenging ${ }^{32}$ but crucial to breaking symmetry for active propulsion. (iii) Fuel molecules can be triggered to realize controlled initiation of propulsion.

Asymmetric geometry of vehicles is important for breaking symmetry to achieve self-propelling behavior. Micro- and nanovehicles experience drag force in biological environments due to the high viscosity of biological media. In this case, Brownian motion becomes more dominant, and inertia forces are negligible for driving the movement of the vehicles. Therefore, an asymmetric geometry is crucial for asymmetrically accumulating catalytic products to create a net driving force for directed motion. ${ }^{33}$ Such symmetry breaking is usually associated with the anisotropic shape of vehicles or the uneven distribution of a certain reactive element, such as metallic catalysts, enzymes, and field-responsive materials, that leads to inhomogeneous physical properties in the same entity. ${ }^{31,34,35}$ Representative processes for propulsions driven by an asymmetric generation of reaction products are bubble propulsion and self-diffusiophoresis. ${ }^{36}$

Motion regulation is another challenge for nanovehicles. Heat pulses have been used to regulate self-propulsion of micro/nanovehicles in a medium. ${ }^{37}$ Velocity of vehicles facing an increased temperature increased significantly compared to that of vehicles kept at room temperature, which was related to the reduced medium's viscosity and temperature dependence of electrochemical consumption of fuel. ${ }^{38}$ Moreover, the combination of a highly reversible process of heat pulses with additional magnetic guidance provided a temporal and spatial control of the velocity and direction of the vehicles. ${ }^{39}$ Recently, Wilson's group successfully built a speed-regulation mechanism on self-assembled stomatocyte nanovehicles. ${ }^{30} \mathrm{~A}$ temperature-sensitive polymer brush was chemically grafted onto nanovehicles to control the opening of stomatocytes upon temperature change, which thus controlled the accessibility of hydrogen peroxide fuel. This, in turn, regulated the movement of the vehicles. Light is another promising tool for motion regulation of vehicles, due to the remote propagation, wireless control, precisely adjustable energy input, and reversible ON/OFF radiation state. ${ }^{40,41}$ Various types of light-driven micro/nanovehicles were developed such as nanobottle motors, ${ }^{42}$ molecular motors, ${ }^{43,44}$ liquid droplets, ${ }^{45}$ solid materials, ${ }^{46-48}$ and DNA-based motors. ${ }^{49-51}$ Propulsion of light-driven motors was mainly reported based on the photothermal effect. ${ }^{52}$ Typically, hybrid Janus particles (e.g., gold-silica or gold-polymer particles displaying distinct faces that absorb light in different amounts) were fabricated. Under light irradiation, a temperature gradient is created near the particle, and the resultant thermal diffusion of the fluid leads to the propulsion of particles. In other examples, ultrafast rotation of gold nanorods was achieved through optical torques from tunable resonant light scattering. However, no clear effect of structural asymmetries on the translational Brownian motion of the rotating nanorods could be observed. ${ }^{53}$

Herein, we developed a one-step approach for the fabrication of silica nanovehicles (SNVs) with an asymmetric head-tail shape and internally stored energy molecules that 
can be initiated by heat and light irradiation. The nanovehicles were designed to mimic the situation in real current vehicles, which carry the fuel that can be initiated to provide the driving force for motion. Cargoes, such as magnetic nanoparticles, were encapsulated in the core-shell structure. Specific focuses of this study are (1) the asymmetric shape control of SNV and its anisotropic growth mechanism, (2) stimuli-responsive gas generation from loaded fuel molecules, and (3) characterization of enhanced diffusion of the nanovehicles.

\section{RESULTS AND DISCUSSION}

Synthesis of Asymmetric Silica Nanovehicles. Silica nanovehicles with a core-shell structure were synthesized in an oil-in-water miniemulsion via a confined sol-gel process of alkoxysilanes at the nanodroplet/water interface (Figure 1a). The oil phase consisted of a mixture of tetraethyl orthosilicate (TEOS) as the silica precursor, hexadecane as the co-stabilizer, chloroform as the solvent, and functional agents to be loaded. The obtained hydrophobic mixture was emulsified with an aqueous solution of cetyltrimethylammonium chloride (CTAC) through a microfluidization process. Here, we used a microfluidic homogenizer (Figure S1), in which the flows of pre-emulsified mixture were passed at a high velocity through an interaction chamber where high impact energy and shear stress were applied on the droplets in order to produce fine emulsions. ${ }^{54,55}$ A detailed procedure of the microfluidization process is described in the Experimental Section. TEOS spontaneously hydrolyzed to silanols by reacting with water at the oil-water interface. Because the experimental $\mathrm{pH}(4-5)$ is above the isoelectric point of silica $(\mathrm{pH} 2-3)$, the hydrolyzed TEOS molecules are negatively charged. ${ }^{56}$ The cationic surfactant played two roles. First, it stabilized the miniemulsion droplets against coalescence. Second, it acted as a templating agent for confining the condensation of hydrolysis products at the interface. A silica shell was hence formed around the nanodroplets via cooperative self-assembly of negatively charged silica species with cationic surfactant. ${ }^{56}$ In this way, secondary nucleation of silica in the continuous phase was avoided. ${ }^{57}$ Hexadecane, due to its very low solubility in water, was used as an osmotic pressure agent to hinder Ostwald ripening of the nanodroplets in order to obtain nanocapsules with a narrow size distribution. ${ }^{58}$

Well-defined core-shell morphologies were identified by using transmission electron microscopy (TEM), indicating the success of confined condensation of alkoxysilanols at the oilwater interface. As shown in Figure $1 b-d$, the asymmetric structure of nanovehicles was controlled by the composition of nanodroplets. Spherical nanocapsules were obtained with a TEOS percentage of $63 \mathrm{wt} \%$ in the droplets (Figure $1 \mathrm{~b}$ ). As the content of TEOS increased, asymmetric morphologies were observed (Figure 1c,d). These asymmetric nanostructures presented two distinct parts: a spherical tank with a large interior cavity and a tail grown out from the nanotank. As the TEOS amount increased to $80 \mathrm{wt} \%$ of the droplets, nanocapsules with mushroom-like shape were formed (Figure 1c). Furthermore, when the nanodroplets consisted of only TEOS (98 wt \%) and hexadecane (2 wt \%) without organic solvent, nanonails with a longer tail were obtained (Figure 1d). The degree of asymmetry of nanocapsules was defined as $A_{s}$ $\left(A_{s}=l / d\right)$ in Figure 1e, where $l$ represents the length of the whole nanostructure (head + tail) and $d$ is the width of the head. When the TEOS percentage in the dispersed phase increased from $63 \mathrm{wt} \%$ to 80 and $98 \mathrm{wt} \%$, the average $A_{\mathrm{s}}$ value of corresponding nanovehicles increased from 1.0 to 1.4 and 2.1 , respectively.

The anisotropic growth mechanism of silica nanocapsules was attributed to the osmotic pressure from ethanol produced by the sol-gel process (Figure 1a). A significant amount of ethanol was produced from the hydrolysis of TEOS (Figure 1f,g). For example, when TEOS accounted for $63 \mathrm{wt} \%$ of the dispersed phase, ethanol represented $56 \mathrm{wt} \%$ of the dispersed phase after condensing the silanol molecules. The partition of ethanol $(1.15 \mathrm{~g})$ in chloroform $(0.7 \mathrm{~g})$ and water $(20 \mathrm{~g})$ was determined to be 9:91 by ${ }^{1} \mathrm{H}$ NMR spectroscopy. After the miniemulsion droplets formed, TEOS started to hydrolyze upon contact with water (Figure 1f,g). The size of the droplets was then fixed due to the formation of the initial silica layers. Subsequently, the continuous conversion of TEOS from inside the droplet results in a solid silica shell and a replacement of the TEOS volume by the generated ethanol (Table S1). The concentration gradient of ethanol between the interior of droplets and the aqueous environment produced an osmotic pressure, which led to an inward diffusion of water across the boundary of the droplet. The inner hydrophobic solution was gradually extruded out of the droplet, which drove the anisotropic deformation of the nanocapsules. In this step, a large amount of solvents was transferred to the aqueous phase via the weaker region of the initial silica shell, thereby transporting TEOS to a new interface created by the flux. TEOS was hydrolyzed upon contact with water. This new interface was stabilized by the Marangoni effect, that is, by the transport of surfactant molecules from the initial droplet/water interface to the deformed new interface. The creation of one hole per nanoparticle by fast sublimation of solvent from polymer nanoparticles containing solvent was also observed. ${ }^{59}$ Bottle-shape carbonaceous ${ }^{42,60}$ and silica ${ }^{17}$ nanoparticles have been reported by other groups based on polymerizationinduced extruding mechanisms. In both cases, an inward diffusion of monomers from continuous phase and subsequent polymerization along the inner surface of the droplets reduced the inner volume for the liquids and thus caused an outflow of inner liquid, forming a fresh template surface for further polymerization.

To confirm this hypothesis, the ratio of TEOS to chloroform in the dispersed phase was varied. With increasing TEOS content, larger amounts of ethanol were produced. Higher osmotic pressure yielded an increased asymmetry (Figure 1bd). A similar trend related to TEOS concentration-dependent formation of asymmetric nanocapsules was observed when using an ultrasonicator for the emulsification (Figure S2), showing the independent influence of TEOS content on the morphology. The asymmetry degree of resulting nanocapsules was larger by using microfluidization in comparison with ultrasonication. This increased asymmetry was due to the deformation of thin silica shell at an early stage induced by the shearing force in the microfluidization process. ${ }^{54}$ Notably, at a TEOS concentration of $63 \mathrm{wt} \%$ in the droplet, no asymmetric nanocapsules were formed using the microfluidizer (Figure S2d). This result shows that only shearing force is not sufficient for forming asymmetric morphology.

The use of organic solvent present in the dispersed phase also influences the formation of asymmetric structures. Chloroform, dichloromethane, $m$-xylene, or oleic triglyceride (with $63 \mathrm{wt} \%$ TEOS) were emulsified in water by ultrasonication. Asymmetric deformation $\left(A_{\mathrm{s}}=1.4\right)$ was only observed in the case of dichloromethane (Figure S3a-d). Two 


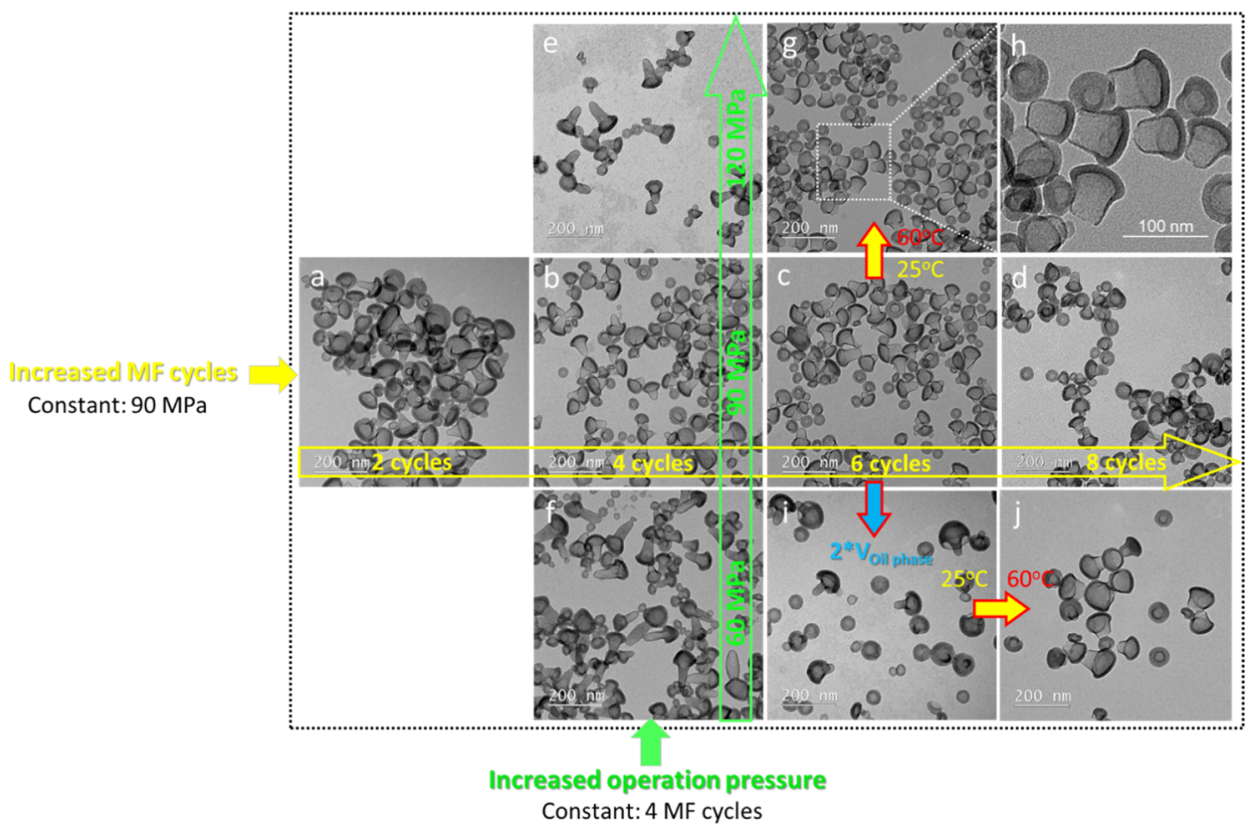

Figure 2. Correlation between microfluidization (MF) processing parameters and morphology of resulting nanocapsules. (a-d) Yellow arrow: nanocapsules prepared with increased $(2-8)$ microfluidizing cycles under constant operation pressure of $90 \mathrm{MPa}$. (e,b,f) Green arrow: nanocapsules prepared under increased operation pressure $(60-120 \mathrm{MPa})$ with four operation cycles. Based on the same miniemulsion as sample (c), "opened" nanocapsules $(\mathrm{g}, \mathrm{h})$ were prepared by heating the miniemulsion at $60{ }^{\circ} \mathrm{C}$ for the sol-gel process. $(\mathrm{i})$ Nanocapsules prepared by using double volume of dispersed phase for the emulsion compared with sample (c). (j) Sample prepared by heating the same miniemulsion as in the case of $(i)$ at $60^{\circ} \mathrm{C}$ after miniemulsification.

possible reasons were hence considered. One hypothesis is that the organic solvent influences the partition coefficient of produced ethanol in the two phases of emulsion. Thus, we measured the partition coefficient of ethanol between oil and water phases for chloroform and dichloromethane by ${ }^{1} \mathrm{H}$ NMR spectroscopy (Figure S4). The results showed similar partition percentages of ethanol in chloroform (22\%) and dichloromethane (23\%), which indicated that ethanol partition was not the reason for this solvent-dependent asymmetric deformation. The second possible reason is the difference of actual volume of the organic solvents in dispersed phase at equilibrium. Because of the higher solubility of dichloromethane in water $(17.5 \mathrm{~g} / \mathrm{L})$ compared to that of chloroform in water $(8.1 \mathrm{~g} / \mathrm{L})$ at $25{ }^{\circ} \mathrm{C}$, the actual volume of dichloromethane in the dispersed phase was calculated to be $70 \%$ of the volume of chloroform. This lower volume, in turn, increased the concentration of TEOS in the nanodroplets, hence being beneficial for the anisotropic growth of nanocapsules as discussed above (Figure 1). The conclusion is that the deformation of nanocapsules depends on the concentration of TEOS in the droplet; asymmetric nanostructures can be formed by using both chloroform and dichloromethane. Anisotropic deformation was further increased to $A_{\mathrm{s}}=1.8$ by using dichloromethane and a microfluidizer for the preparation (Figure S3b2).

Because the microfluidization process was beneficial for the formation of asymmetric shape at the nanoscale, which is crucial for nanovehicles to break symmetry for active propulsion, we investigated how the microfluidizing parameters influence the morphology of nanocapsules. In the microfluidization process, high pressure was applied to produce a flow of pre-emulsified mixture at a high velocity through an interaction chamber. This chamber consists of microchannels with diameters in the range of 50 to $200 \mu \mathrm{m}$.
The flow is divided into separated microstreams, which were subsequently brought together in an impinging geometry to create high impact energy and shear stress on the droplets. ${ }^{54}$ The size of resulting nanodroplets strongly depends on the energy input to the system, which can be controlled by two parameters: (1) the pressure used to propel emulsion stream in the interaction chamber and (2) the number of cycles that the emulsion was passed through the interaction chamber. In the microfluidizer, the pressure applied is transformed into shear and impact forces. These disruptive forces play a key role in overcoming the surface energy and viscoelastic energy of the droplet. To study the effect of pressure, all samples were passed four cycles through the interaction chamber. The results showed that by increasing the processing pressure from 60 to $120 \mathrm{MPa}$, the intensity-averaged hydrodynamic diameter of nanocapsules was reduced by $18 \%$ (images guided by green arrows in Figure 2). The formation of smaller and more uniform nanocapsules at higher processing pressure was due to increased velocity of the fluid in the microchannel when the pressure was increased.

An alternative way to increase the energy input for breaking down the emulsion droplets into nanometer size is to pass the emulsion multiple times through the microfluidizer. Complete hydrolysis of silica precursor occurred in $5 \mathrm{~h}$ (Figure 1f). On the contrary, the whole microfluidizing process took $2-5 \mathrm{~min}$ (for $2-8$ cycles). At this stage, the silica network is not dense, and therefore, droplets with a very thin shell can be deformed upon shearing force. Herein, we kept the operation pressure constant at $90 \mathrm{MPa}$, and the emulsified suspensions were passed through the interaction chamber for $2-8$ cycles (images guided by yellow arrow in Figure 2). As the processing cycles increased, the anisotropy of nanocapsules increased with a clear reduction of the volume of the head compartment (Figure $2 \mathrm{a}-\mathrm{d}$ ). The obtained nanocapsules became more 
(a)

AIBN (fuel)

- $\mathrm{N}_{2}$ bubble

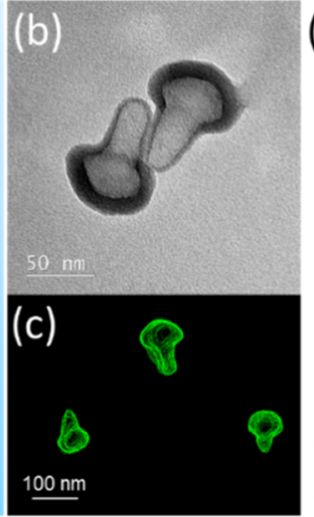

(f)

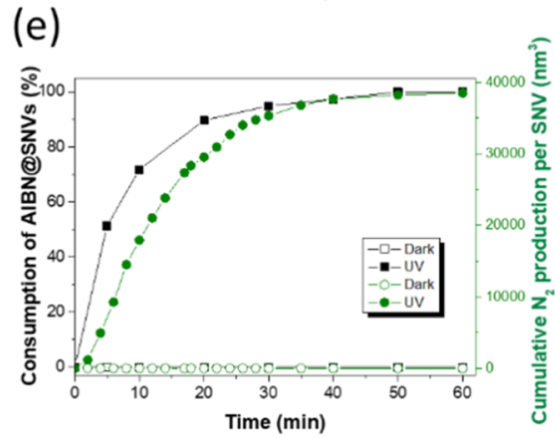

(d)

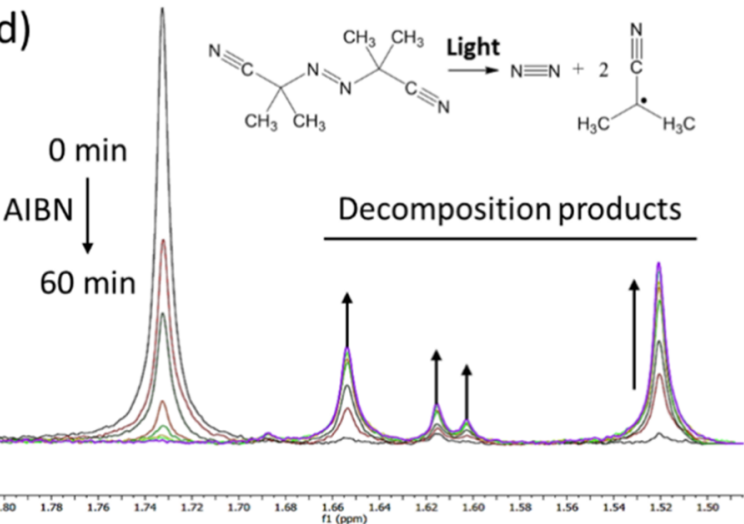

(g)
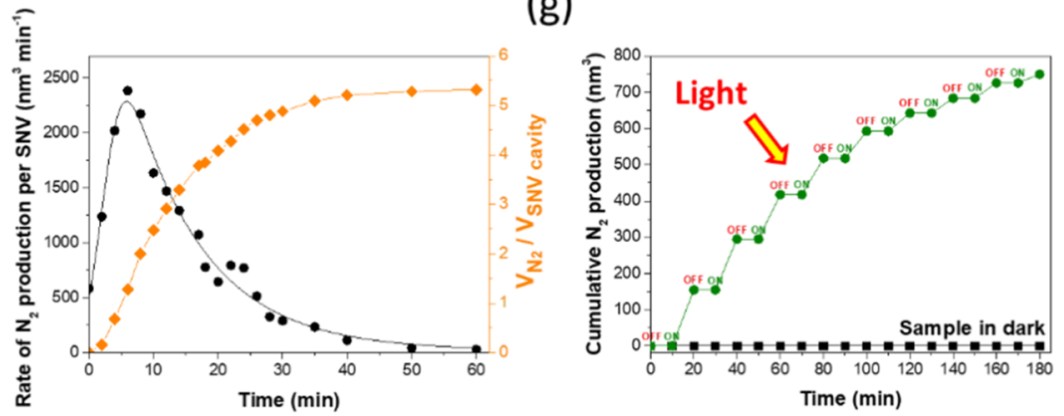

Figure 3. (a) Schematic illustration of the self-propulsion mechanism of fuel-containing nanovehicles. (b) TEM micrograph of asymmetric nanovehicles containing AIBN. (c) Electron tomography reconstruction of the individual nanostructure. (d) Decomposition of AIBN@SNVs under UV irradiation $\left(\lambda=360 \mathrm{~nm}\right.$ ) investigated by ${ }^{1} \mathrm{H}$ NMR spectroscopy. (e) Consumption of AIBN calculated from ${ }^{1} \mathrm{H}$ NMR spectra and cumulative $\mathrm{N}_{2}$ generation per SNV measured with a bubble flowmeter. (f) Rate of $\mathrm{N}_{2}$ generation per $\mathrm{SNV}$ and volume ratio of $\mathrm{N}_{2}$ to $\mathrm{SNV}$ inner cavity. (g) Profile of cumulative $\mathrm{N}_{2}$ production from SNVs during light $\mathrm{ON} / \mathrm{OFF}$ circles.

uniform, as evidenced in TEM images. The hydrodynamic diameter of nanocapsules decreased from $105 \pm 33 \mathrm{~nm}$ (two cycles) to $70 \pm 23 \mathrm{~nm}$ (eight cycles). The morphology of asymmetric nanocapsules can be further controlled by changing the temperature during the sol-gel process. Mushroom-like nanocontainers with an open connection were obtained when the temperature for the sol-gel process was increased from 25 to $60{ }^{\circ} \mathrm{C}$ (Figure $2 \mathrm{~h}, \mathrm{j}$ ), thus forming an open system that allows mass exchange between the interior of the nanocapsules and the environment. This open connection is useful for applications in catalysis. Indeed, asymmetric nanobottles with an open large cavity loaded with platinum and gold nanoparticles were used as catalytic nanomotors and nanoreactors. ${ }^{17}$ Herein, we loaded the interior of nanovehicles with $\mathrm{Fe}_{3} \mathrm{O}_{4}$ nanoparticles ${ }^{30}$ during their formation because of their potential use for magnetic separation or magnetic-guided targeted delivery. The magnetic nanoparticles were clearly identified in the capsules by TEM (Figure S5a). The superparamagnetism of iron oxide nanoparticles was preserved, with a saturated magnetization of $6.0 \mathrm{Am}^{2} / \mathrm{kg}$ (Figure S5b). The magnetic nanocapsules were hence able to be separated from aqueous media by a magnet in $3 \mathrm{~min}$ (Figure S5c). Magnetic-controlled transport and directional motion of nanovehicles are discussed in the Supporting Information (Figures S6-S8 and Video S1).

To understand the effect of temperature on the formation of asymmetric nanocapsules, we studied the morphology of nanocapsules formed at 25 and $60{ }^{\circ} \mathrm{C}$ after emulsification (Figure S9). The width of the nanocapsules' tail increased when heating (at $60{ }^{\circ} \mathrm{C}$ ) was applied immediately after the emulsification ( $t=0 \mathrm{~h}$, Figure S9a). When the heating was delayed to $t=4 \mathrm{~h}$ after emulsification, the tail's width was smaller (Figure S9b). For samples heated at $t=18 \mathrm{~h}$ after the silica formation started, the morphology of the nanocapsule was almost the same as for samples prepared at $25^{\circ} \mathrm{C}$ (Figure S9c,e). The reason was that the core-shell morphology of nanocapsules was already formed after $4 \mathrm{~h}$ (Figure S9d), and further heating did not induce additional deformation. This observation was verified by kinetic study of the sol-gel process by ${ }^{1} \mathrm{H}$ NMR spectroscopy. Indeed, $\sim 80 \%$ TEOS was hydrolyzed in $4 \mathrm{~h}$, and the reaction was completed in $5 \mathrm{~h}$ (Figure 1f).

Light- and Heat-Induced Consumption of Fuels and Propulsion Study of the Nanovehicles. In the next step, fuel molecules were encapsulated in the inner cavity of nanocapsules, named silica nanovehicles (SNVs). Azo-based radical initiators, which release nitrogen gas and generate radical species upon decomposition under heat or UV irradiation, were chosen as the fuel. ${ }^{61}$ Specifically, 2,2'azobis(isobutyronitrile) (AIBN) was first mixed with alkoxysilane and dichloromethane to form the dispersed phase that was then converted to nanodroplets after emulsification. During the sol-gel process of alkoxysilane, the fuel was encapsulated in situ in the core of the nanovehicles (Figure S10). A schematic illustration of the mechanism of self-fueled nanovehicles is shown in Figure 3a. Nanovehicles with a length of $\sim 100 \mathrm{~nm}$ and a head-tail structure were observed by TEM (Figure $3 b$ ). To further identify their internal structure, the nanovehicles were observed by electron tomography (Figure S11). Two-dimensional projections were acquired at different heights and tilting angles. Core-shell structures with a hollow interior were confirmed from the reconstruction of the two- 
(a)

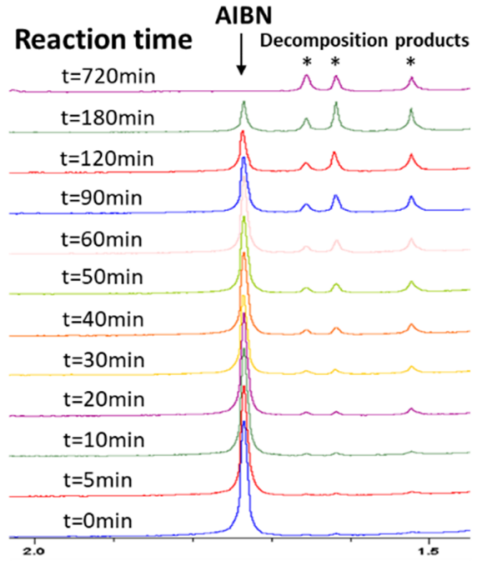

(c)

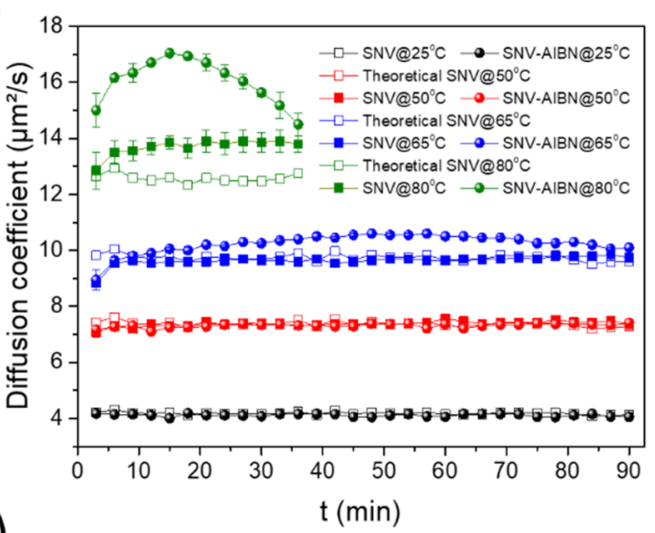

(e)

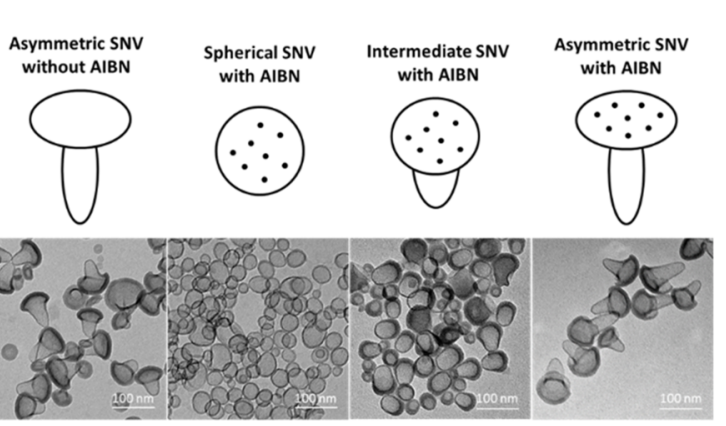

(b)

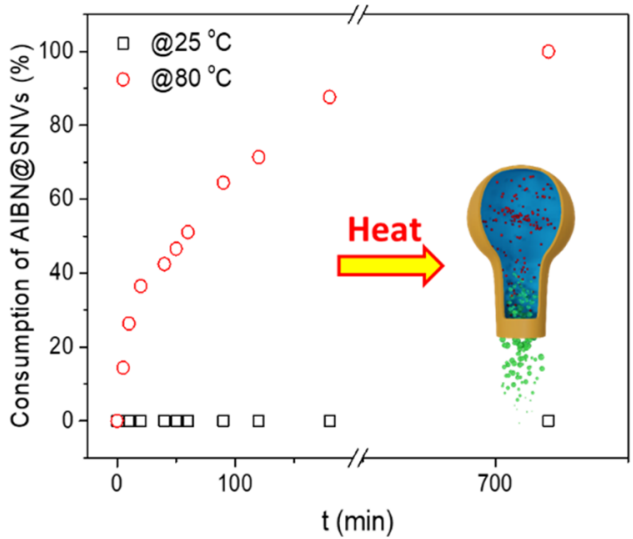

(d)

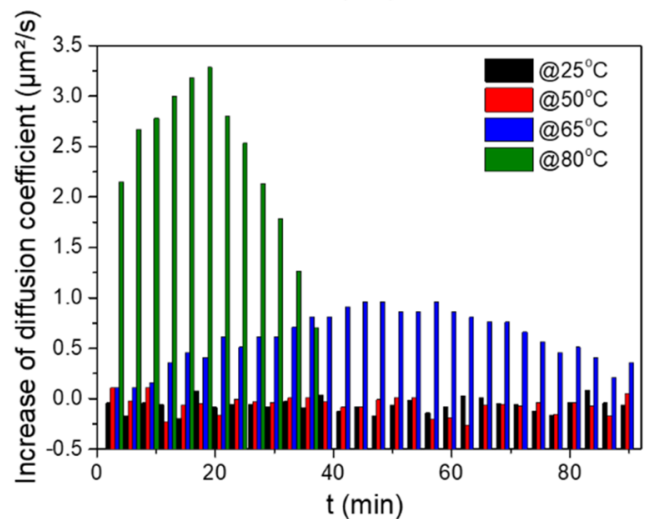

(f)

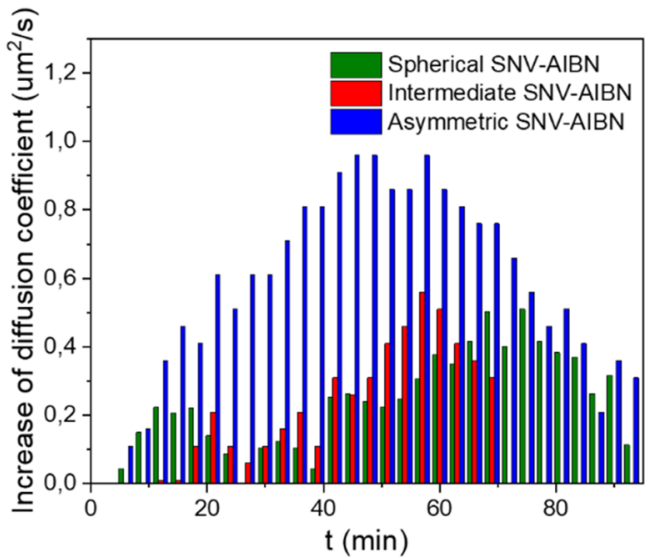

Figure 4. (a) Decomposition kinetics of AIBN@SNVs under heating at $80{ }^{\circ} \mathrm{C}$ investigated by ${ }^{1} \mathrm{H}$ NMR spectroscopy. (b) Consumption of the fuel (AIBN) at 25 and $80{ }^{\circ} \mathrm{C}$ calculated from ${ }^{1} \mathrm{H}$ NMR spectroscopy measurements. (c) Diffusion coefficient of nanovehicles without and with fuel at different temperatures. (d) Increase of diffusion coefficient calculated by subtracting the diffusion coefficient of SNVs with AIBN with SNVs without AIBN. (e) Schematic illustration and TEM micrographs and (f) increase of diffusion coefficient of SNVs with different degrees of asymmetry compared with SNVs in the absence of fuel.

dimensional data (Figure 3c). Nanocapsules containing AIBN are closed. Due to the osmotic pressure-induced deformation mechanism, the shell thickness of the nanocapsule decreases from the head hemisphere to the tail. The tip of the tails is composed of a very thin silica layer which allows an easier escape of generated nitrogen gas.

The concentration of AIBN loaded in nanovehicles was measured to be $1.48 \mathrm{mg} / \mathrm{mL}$ by ${ }^{1} \mathrm{H}$ NMR (Figure S12a), which is close to the weighted amount of AIBN $(1.75 \mathrm{mg} / \mathrm{mL}$ dispersion) before emulsification. Furthermore, AIBN in the aqueous phase of the dispersion subjected to ultrafiltration could not be detected (Figure S12b), hence confirming the efficient encapsulation of fuel molecules in the vehicle. Finally, the effective loading of hydrophobic cargos in the inner cavity of nanovehicles was also supported by the fact that oleic-acidstabilized $\mathrm{Fe}_{3} \mathrm{O}_{4}$ nanoparticles were located inside the core of nanovehicles (Figure S5a).

Subsequently, we investigated the profiles of gas generation from AIBN-loaded nanovehicles in response to light irradiation. Aqueous dispersions of nanovehicles were irradiated by UV light at room temperature. The decomposition of AIBN in nanovehicles was monitored by quantifying the signal related to protons of methyl groups in $\operatorname{AIBN}(\delta=1.73 \mathrm{ppm})$ by ${ }^{1} \mathrm{H}$ NMR spectroscopy. As shown in Figure $3 \mathrm{~d}$, the decrease of signal at $1.73 \mathrm{ppm}$ and increase of signal in the range of 1.68-1.48 ppm indicated the decomposition of AIBN in 
tetramethylsuccinonitrile and other products. ${ }^{61}$ After $1 \mathrm{~h}$, AIBN was completely decomposed. This decomposition was confirmed by Fourier transform infrared (FTIR) spectroscopy. The intensity of the $\mathrm{N}=\mathrm{N}$ stretching bands at $1464 \mathrm{~cm}^{-1}$ decreased after light irradiation (Figure S13). The decomposition of AIBN followed a concentration-dependent (firstorder) kinetics (Figure 3e). Around 50\% AIBN was decomposed in $10 \mathrm{~min}$, whereas the reaction was completed in $1 \mathrm{~h}$. In parallel, the production of nitrogen gas was quantified by using a capillary version bubble flowmeter. The release profile of $\mathrm{N}_{2}$ (green line) was consistent with the decomposition profile of AIBN (black line) monitored by ${ }^{1} \mathrm{H}$ NMR spectroscopy (Figure 3e). The small delay in the case of $\mathrm{N}_{2}$ production was likely due to the low sensitivity of the flowmeter for small gas volumes. Rate of nitrogen production was found to fit a first-order derivative. The maximum rate occurred at $6 \mathrm{~min}$, and the final volume of generated nitrogen gas under ambient pressure at $25{ }^{\circ} \mathrm{C}$ was 5 times larger than the volume of the inner cavity of the nanovehicles (Figure 3f). Light has the advantage of providing a wireless remote control on motion, and it is possible to precisely adjust the energy input to the nanovehicles. As shown in Figure $3 \mathrm{~g}$, the production of $\mathrm{N}_{2}$ can be reversibly triggered and suppressed in response to the light radiation state.

Thermally induced decomposition of AIBN in nanovehicles was monitored by ${ }^{1} \mathrm{H}$ NMR spectroscopy by heating the nanovehicles at $80{ }^{\circ} \mathrm{C}$. As shown in Figure $4 \mathrm{a}$, the signal of AIBN at $1.73 \mathrm{ppm}$ decreased with time, and new signals for the decomposition products appeared in the range of 1.68-1.48 ppm. The heat-induced decomposition of AIBN followed concentration-dependent kinetics similar to those for the lightinduced activation (Figure 4b). However, the decomposition rate was slower in the case of thermally induced decomposition at $80{ }^{\circ} \mathrm{C}$. Indeed, around $50 \%$ of AIBN was decomposed in the first hour. After 3 h, $90 \%$ of the AIBN was decomposed and complete decomposition was observed after $12 \mathrm{~h}$.

Motion of micro- and nanovehicles is often observed by optical microscopy. Mean-squared displacements of vehicles at different time intervals are typically calculated based on a statistical analysis of their trajectories. This calculation enables differentiation between self-propulsion and other motions such as sedimentation, convection, and Brownian motion. ${ }^{36,62}$ However, when particles become smaller, Brownian motion becomes more dominant and results in fast reorientation of the nanoparticles, making direct measurements of their velocities experimentally challenging. ${ }^{63}$ Alternatively, self-propulsion of nanovehicles can be characterized as enhanced diffusion. Indeed, propelled nanovehicles shall diffuse with an effective diffusion coefficient $D_{\text {eff }}$ higher than its equilibrium value $D_{0}$ in the absence of directed motion. ${ }^{36}$ Herein, the $D_{\text {eff }}$ of nanovehicles was measured at temperatures ranging from 25 to $80{ }^{\circ} \mathrm{C}$ by dynamic light scattering. Nanovehicles without AIBN, acting as control samples, were measured in the same way as $D_{0}$. Theoretical diffusion coefficients $D_{\text {th }}$ at 50,65 , and $80{ }^{\circ} \mathrm{C}$ were also calculated with the Stokes-Einstein equation (Figure S14) based on $D_{0}$ measured at $25{ }^{\circ} \mathrm{C}$. The StokesEinstein equation is only valid for hard spheres, but there was no appropriate model for the coefficient of friction of our particles. Models for rod-shaped and ellipsoids take into account the geometric features of the diffusing objects. However, the temperature dependency in the relationship between diffusion coefficient and geometric values is the same. Importantly, the measured $D_{0}$ values at 50,65 , and $80{ }^{\circ} \mathrm{C}$ are consistent with the calculated $D_{\text {th }}$ at respective temperatures (Figure 4c), showing the reliability of the diffusion coefficient measurements. With AIBN encapsulated in the SNVs, there was no significant increase of diffusion occurring in $90 \mathrm{~min}$ at 25 and $50{ }^{\circ} \mathrm{C}$ (Figure 4c). AIBN started to decompose when the temperature was increased to $65{ }^{\circ} \mathrm{C},{ }^{64}$ and a gradual increase of $D_{\text {eff }}$ was observed in comparison to the control sample in the same experimental condition. Up to $12 \%$ increase in $D_{\text {eff }}$ was achieved at $\sim 48 \mathrm{~min}$. Afterward, $D_{\text {eff }}$ started to decrease due to the consumption of fuel. Furthermore, enhanced diffusion with an increase of 3.3 $\mu \mathrm{m}^{2} / \mathrm{s}$ in $D_{\text {eff }}$ was achieved in $18 \mathrm{~min}$ as the temperature was increased to $80^{\circ} \mathrm{C}$ (Figure $4 \mathrm{c}, \mathrm{d}$ ). This temperature-dependent acceleration of diffusion was attributed to the increased decomposition rate of an azo-based initiator at higher temperature. ${ }^{65}$ Indeed, $\sim 37 \%$ of AIBN was decomposed in the first $18 \mathrm{~min}$ of heating at $80{ }^{\circ} \mathrm{C}$ (Figure $4 \mathrm{~b}$ ).

Delay of maximum increase of $D_{\text {eff }}$ at different temperatures was observed in Figure 4d, which can be explained by two reasons. First, the amount of generated nitrogen molecules is initially low and therefore can diffuse isotropically through the silica membrane before saturation is achieved. Thus, no directional movement was detected at initial stage. About $25 \%$ of nitrogen gas was produced before reaching the maximum $D_{\text {eff }}$ at $15 \mathrm{~min}$ at $80{ }^{\circ} \mathrm{C}$ (Figure $4 \mathrm{~b}, \mathrm{c}$ ). This also explains the short delay at $80{ }^{\circ} \mathrm{C}$ because of the fast generation rate of nitrogen compared to the longer delay at $65{ }^{\circ} \mathrm{C}$. Second, decomposition of AIBN is an exothermic reaction. The continuous production of heat could increase local temperature, which accelerates the decomposition reaction and forms a thermal flow from the interior of the capsule to the surroundings, providing hydrodynamic stresses necessary to overcome viscous resistance and to induce propulsion.

In fact, the increase of diffusion coefficient $\left(D_{\text {eff }}\right)$ is correlated with the conversion percentage and decomposition rate of AIBN (Figure S15). We calculated the decomposition rate of AIBN by differentiating the conversion kinetic at $80{ }^{\circ} \mathrm{C}$. The initial decomposition rate of AIBN@SNVs is relatively fast, which induced a rapid enhancement of particle diffusion. The $\Delta D_{\text {eff }}$ increased slightly and was maintained at $2-3 \mu \mathrm{m}^{2} / \mathrm{s}$ at the decomposition rate over $0.5 \% / \mathrm{min}$. Diffusion started to slow down as the AIBN content and decomposition rate further decreased. At $65{ }^{\circ} \mathrm{C}$, due to the relatively longer lifetime of AIBN, the duration for which $D_{\text {eff }}$ increases was larger (Figure 4d).

To study the correlation between enhanced diffusion and fuel consumption, $\Delta D_{\text {eff }}$ was plotted against the conversion percentage and decomposition rate of AIBN@SNVs. As shown in Figure S16a, $\Delta D_{\text {eff }}$ increased with conversion of AIBN at the initial stage. At conversion over $\sim 36 \%, \Delta D_{\text {eff }}$ started to decrease due to the consumption of AIBN. As shown in Figure $\mathrm{S} 16 \mathrm{~b}$, the increase of diffusion coefficient was positively correlated with the rate of AIBN consumption. As the conversion rate increased, $\Delta D_{\text {eff }}$ first increased linearly and then remained constant at around $3 \mu \mathrm{m}^{2} / \mathrm{s}$.

To further investigate the correlation between nanoparticle morphology and their diffusion coefficient, a series of nanovehicles with shapes ranging from spherical to asymmetric were prepared (Figure $4 \mathrm{e}$ ). Their $D_{\text {eff }}$ was compared with the $D_{0}$ of asymmetric SNV without fuel in the same conditions. As shown in Figure S17, without AIBN, the asymmetric SNVs showed a stable diffusion with $D_{0}$ of $9.5-9.7 \mu \mathrm{m}^{2} / \mathrm{s}$. Increases of diffusion coefficient were observed for all nanovehicles with 
encapsulated fuel at $65^{\circ} \mathrm{C}$ (Figure 4f). Particularly, asymmetric SNVs containing AIBN and with a higher asymmetric degree showed the highest $D_{\text {eff }}$ compared with SNVs with a spherical shape or a lower degree of asymmetry. These results indicated that the asymmetric geometry of nanovehicles is beneficial for achieving more efficient self-propelling behavior due to the asymmetric accumulation of reaction products at one end of the vehicle, which forms the net driving force to achieve selfpropellancy. An increased ratio of length to radius (in the range of $0-3$ ) was reported to significantly reduce drag coefficient of micro/nanomotors with a cone-frustum shape in a fluid. ${ }^{66}$ Because the SNVs with different asymmetries contained the same amount of AIBN, the possible interference of bubble generation on light scattering was excluded for this enhanced diffusion. The asymmetric geometry also influences the distribution gradient of reaction products, which was systematically studied for Janus catalytic particles. ${ }^{67}$ It was found that the geometry of the particle is decisive for the selfpropulsion due to the different distribution of product gradient determined by the geometrical differences of the two faces of the particle. Opposite swimming directions (e.g., away from $^{68,69}$ and toward ${ }^{70}$ the catalytic site) have been observed in experiments for swimmers with identical surface and chemical properties and chemical conditions but with a different geometry.

\section{CONCLUSIONS}

Here, we designed self-propelled nanovehicles with internally stored fuels so that their locomotion can be activated by external stimuli. The nanovehicles were prepared in a one-pot process via a confined sol-gel process at the oil/water interface of miniemulsion droplets. Nanovehicles displayed shapes ranging from mushroom-like to truncated cones. The asymmetric geometry of vehicles was important for breaking symmetry to achieve self-propelling behavior. The core-shell structure was designed so that the silica shell acted as a hull for the nanovehicles while the core was used to store the fuel. Azobased initiators were loaded in the nanovehicles, which were activated to release nitrogen gas upon increase of temperature or exposure to UV light. Enhanced diffusion of nanovehicles was achieved upon decomposition of the fuel. These active nanovehicles are promising for implementing additional functions to future nanobots.

Although significant progress has been achieved in the past decades, challenges are emerging as the dimensions of vehicles and their motion drop to nanometer scale. These interesting issues include (i) facile synthesis of nanosized vehicles via bottom-up approaches that can be easily scaled up and avoids biocompatibility issues; (ii) control of asymmetry at nanoscale by forming an anisotropic shape of vehicles or through the uneven distribution of catalysts, which is crucial for asymmetrically accumulating catalytic products to create a net driving force for directed motion; (iii) development of nanomotors that use existing substrates (e.g., water and glucose) from their surroundings, more interestingly, without the need of external fuels; (iv) regulating the movement and directionality of nanovehicles through external stimuli; (v) direct measurement of the velocities of nanosized vehicles by optical microscopes, which is experimentally challenging because nano-objects usually move in three-dimensional volumes even in very thin liquid films. Motions along the $Z$ axis direction are difficult to be quantified. These questions require more sophisticated material design as well as the development of advanced controlling and characterization methods for the motion of nanoparticles.

\section{EXPERIMENTAL SECTION}

Synthesis of Asymmetric Silica Nanocapsules. Silica nanocapsules were synthesized in oil-in-water miniemulsions using the surface of oil nanodroplets as a template for the hydrolysis and condensation of alkoxysilanes. Specifically, $2.0 \mathrm{~g}(9.6 \mathrm{mmol})$ of TEOS was first mixed with $125 \mathrm{mg}$ of hexadecane and $1 \mathrm{~g}$ of chloroform or dichloromethane to form the oil phase. In the second step, $30 \mathrm{~mL}$ of $0.77 \mathrm{mg} \cdot \mathrm{mL}^{-1}$ aqueous solution of cationic surfactant CTAC was poured into the oil solution under stirring at $1000 \mathrm{rpm}$. The oil/water mixture was homogenized with a T25 Ultra-Turrax at $13000 \mathrm{rpm}$ for $1 \mathrm{~min}$. The emulsion was then injected in a microfluidizer (LM10, Microfluidics Corporation) cooled with ice. The microfluidizer processor was equipped with an air-driven intensifier pump that supplied the desired pressure at a constant rate to the emulsion stream. The pressure was controlled digitally via the user interface. The processed emulsions were collected from the outlet flow and could be fed to the inlet reservoir for multiple circle processing (Figure S1). In order to tune the size and asymmetric structure of formed nanovehicles, operating pressure $(60-120 \mathrm{MPa})$ and cycles $(2-8)$ that the emulsion was processed through the microfluidizer were varied. The resulting miniemulsions were stirred at $1000 \mathrm{rpm}$ for $12 \mathrm{~h}$ at $25{ }^{\circ} \mathrm{C}$ to allow the hydrolysis and condensation of the silica precursor and the asymmetric growth of nanoparticles. To prepare nanocapsules with opened tails, the miniemulsion after microfluidization was stirred at $60{ }^{\circ} \mathrm{C}$ under $1000 \mathrm{rpm}$ for $12 \mathrm{~h}$. For the self-propulsion study, nanovehicles containing fuel were synthesized by dissolving $60 \mathrm{mg}$ of AIBN in the oil phase. For magnetic-controlled motion, $160 \mathrm{mg}$ of oleic-acid-stabilized $\mathrm{Fe}_{3} \mathrm{O}_{4}$ nanoparticles was dissolved in the oil phase. For fluorescent labeling of the nanovehicles, Cy5-NHS ester was first coupled with APTES at a molar ratio of 1:1.1 to obtain fluorescently labeled silica precursors. The APTES-Cy5 conjugates were then mixed with TEOS as the silica source. The molar ratio of Cy5 with TEOS was 1:14000. All of the emulsions for the self-propulsion study were processed under a pressure of $90 \mathrm{MPa}$ for four cycles. Samples were purified by centrifugation in water to remove excess surfactant.

Analytical Tools. Hydrodynamic diameters of nanoparticles were measured by dynamic light scattering (Malvern S90) at a fixed scattering angle of $90^{\circ}$. The morphology of nanoparticles was examined with a JEOL 1400 (JEOL Ltd., Tokyo, Japan) transmission electron microscope operating at an accelerating voltage of $120 \mathrm{kV}$. TEM samples of nanocapsules were prepared by casting the diluted dispersions on carbon-layer-coated copper grids. The magnetic property of nanovehicles was determined with a superconducting quantum interference device (SQUID) magnetometer (Quantum Design MPMS XL). Nanovehicles loaded with $\mathrm{Fe}_{3} \mathrm{O}_{4}$ nanoparticles were freeze-dried and then collected in gelatin capsules, which provided a negligible purely diamagnetic background signal in the SQUID measurement. The SQUID curve was measured at $300 \mathrm{~K}$. The tomographic tilt series of nanoparticles were acquired using a FEI Tecnai F20 transmission electron microscope, operated at an accelerating voltage of $200 \mathrm{kV}$. The samples were tilted over a $120^{\circ}$ range at $1^{\circ}$ interval. The scale in each image is $\sim 0.36 \mathrm{~nm}$ per pixel. From these projections in the tilt series, the final reconstruction was created using SerialEM package software. Solid content of the particle dispersion was measured gravimetrically. The samples were freezedried at 0.1 mbar pressure overnight. ${ }^{1} \mathrm{H}$ NMR spectra were recorded using a Bruker Avance 300 or a Bruker Avance 850. FTIR spectra were recorded by using a spectrum BX spectrometer (PerkinElmer). Nanoparticle dispersions were freeze-dried, and the powders were pressed with $\mathrm{KBr}$ to form a pellet. Transmittance between 4000 and $500 \mathrm{~cm}^{-1}$ was recorded. To calculate the degree of asymmetry, the length and diameter of asymmetric nanoparticles were measured by using software ImageJ. Average results of 100 objects in TEM micrographs were reported. The volume of produced nitrogen gas under UV irradiation (Osram Mercury lamp, HBO 100W/2) was 
measured by using a bubble flowmeter (capillary version, SigmaAldrich). Diffusion coefficient $(D)$ of the nanovehicles was measured by dynamic light scattering using a Malvern Zeta-Sizer (Nano S90, Malvern Instruments, UK). The measurements were performed at a fixed scattering angle of $90^{\circ}$. The diffusion coefficient was monitored continuously with time at a fixed temperature. The temperature was varied from 25 to 50,65 , and $80{ }^{\circ} \mathrm{C}$. Average results from three experiments were reported.

\section{ASSOCIATED CONTENT}

\section{SI Supporting Information}

The Supporting Information is available free of charge at https://pubs.acs.org/doi/10.1021/acsnano.9b06408.

Materials; synthesis of $\mathrm{Fe}_{3} \mathrm{O}_{4} \mathrm{NPs}$; image of microfluidizer; magnetic controlled motion of nanovehicles; additional figures including TEM micrographs of SNVs prepared with various techniques, solvents, amounts of TEOS, and temperatures; ${ }^{1} \mathrm{H}$ NMR spectra of ethanol and AIBN; TEM micrographs, SQUID magnetization curve, photograph of magnetic separation of iron-oxidelabeled SNVs; schematic illustration of miniemulsion polymerization process; electron tomography of $\mathrm{SNV}$; FTIR spectra of AIBN; calculation for theoretical diffusion coefficient of SNVs at different temperatures; diffusion coefficient of SNVs with different degrees of asymmetry and in the absence and presence of fuel (PDF)

Video S1: magnetic directed motion (AVI)

\section{AUTHOR INFORMATION}

\section{Corresponding Authors}

Daniel Crespy - Vidyasirimedhi Institute of Science and Technology (VISTEC), Rayong, Thailand; (D orcid.org/ 0000-0002-6023-703X; Email: daniel.crespy@vistec.ac.th

Katharina Landfester - Max Planck Institute for Polymer Research, Mainz, Germany; (1) orcid.org/0000-0001-95914638; Email: landfester@mpip-mainz.mpg.de

\section{Other Authors}

Shuai Jiang - Max Planck Institute for Polymer Research, Mainz, Germany, and Vidyasirimedhi Institute of Science and Technology (VISTEC), Rayong, Thailand

Anke Kaltbeitzel - Max Planck Institute for Polymer

Research, Mainz, Germany

Minghan Hu - Max Planck Institute for Polymer

Research, Mainz, Germany; (1) orcid.org/0000-0002-90016780

Oksana Suraeva - Max Planck Institute for Polymer

Research, Mainz, Germany

Complete contact information is available at:

https://pubs.acs.org/10.1021/acsnano.9b06408

\section{Notes}

The authors declare no competing financial interest.

\section{ACKNOWLEDGMENTS}

We acknowledge Stefan Schuhmacher for the design of the schemes, Mr. Henrik Schmidt for helping with the magnetic motion of the nanoparticles, and Dr. Kyujoon Lee for the SQUID measurement. D.C. was supported by the Office of the Higher Education Commission of Thailand (OHEC) and the
Vidyasirimedhi Institute of Science and Tehcnology (VISTEC).

\section{REFERENCES}

(1) Wang, H.; Khezri, B.; Pumera, M. Catalytic DNA-Functionalized Self-Propelled Micromachines for Environmental Remediation. Chem. 2016, 1, 473-481.

(2) Gao, W.; Wang, J. The Environmental Impact of Micro/ Nanomachines: A Review. ACS Nano 2014, 8, 3170-3180.

(3) Vilela, D.; Parmar, J.; Zeng, Y.; Zhao, Y.; Sánchez, S. GrapheneBased Microbots for Toxic Heavy Metal Removal and Recovery from Water. Nano Lett. 2016, 16, 2860-2866.

(4) Wang, J. Self-Propelled Affinity Biosensors: Moving the Receptor around the Sample. Biosens. Bioelectron. 2016, 76, 234-242.

(5) Moo, J. G. S.; Pumera, M. Self-Propelled Micromotors Monitored by Particle-Electrode Impact Voltammetry. ACS Sensors 2016, 1, 949-957.

(6) Baraban, L.; Makarov, D.; Streubel, R.; Monch, I.; Grimm, D.; Sanchez, S.; Schmidt, O. G. Catalytic Janus Motors on Microfluidic Chip: Deterministic Motion for Targeted Cargo Delivery. ACS Nano 2012, 6, 3383-3389.

(7) Gao, W.; Kagan, D.; Pak, O. S.; Clawson, C.; Campuzano, S.; Chuluun-Erdene, E.; Shipton, E.; Fullerton, E. E.; Zhang, L.; Lauga, E.; et al. Cargo-Towing Fuel-Free Magnetic Nanoswimmers for Targeted Drug Delivery. Small 2012, 8, 460-467.

(8) Patra, D.; Sengupta, S.; Duan, W.; Zhang, H.; Pavlick, R.; Sen, A. Intelligent, Self-Powered, Drug Delivery Systems. Nanoscale 2013, 5, 1273-1283.

(9) Tu, Y.; Peng, F.; André, A. A.; Men, Y.; Srinivas, M.; Wilson, D. A. Biodegradable Hybrid Stomatocyte Nanomotors for Drug Delivery. ACS Nano 2017, 11, 1957-1963.

(10) Lee, T.-C.; Alarcón-Correa, M.; Miksch, C.; Hahn, K.; Gibbs, J. G.; Fischer, P. Self-Propelling Nanomotors in the Presence of Strong Brownian Forces. Nano Lett. 2014, 14, 2407-2412.

(11) Ma, X.; Hahn, K.; Sanchez, S. Catalytic Mesoporous Janus Nanomotors for Active Cargo Delivery. J. Am. Chem. Soc. 2015, 137, 4976-4979.

(12) Kagan, D.; Laocharoensuk, R.; Zimmerman, M.; Clawson, C.; Balasubramanian, S.; Kang, D.; Bishop, D.; Sattayasamitsathit, S.; Zhang, L.; Wang, J. Rapid Delivery of Drug Carriers Propelled and Navigated by Catalytic Nanoshuttles. Small 2010, 6, 2741-2747.

(13) Patiño, T.; Feiner-Gracia, N.; Arqué, X.; Miguel-López, A.; Jannasch, A.; Stumpp, T.; Schäffer, E.; Albertazzi, L.; Sanchez, S. Influence of Enzyme Quantity and Distribution on the SelfPropulsion of Non-Janus Urease Powered Micromotors. J. Am. Chem. Soc. 2018, 140, 7896-7903.

(14) Esteban-Fernández de Ávila, B.; Martín, A.; Soto, F.; LopezRamirez, M. A.; Campuzano, S.; Vásquez-Machado, G. M.; Gao, W.; Zhang, L.; Wang, J. Single Cell Real-Time miRNAs Sensing Based on Nanomotors. ACS Nano 2015, 9, 6756-6764.

(15) Ma, X.; Jannasch, A.; Albrecht, U.-R.; Hahn, K.; Miguel-López, A.; Schäffer, E.; Sánchez, S. Enzyme-Powered Hollow Mesoporous Janus Nanomotors. Nano Lett. 2015, 15, 7043-7050.

(16) Keller, S.; Teora, S. P.; Hu, G. X.; Nijemeisland, M.; Wilson, D. A. High-Throughput Design of Biocompatible Enzyme-Based Hydrogel Microparticles with Autonomous Movement. Angew. Chem., Int. Ed. 2018, 57, 9814-9817.

(17) Yi, D.; Zhang, Q.; Liu, Y.; Song, J.; Tang, Y.; Caruso, F.; Wang, Y. Synthesis of Chemically Asymmetric Silica Nanobottles and Their Application for Cargo Loading and as Nanoreactors and Nanomotors. Angew. Chem., Int. Ed. 2016, 55, 14733-14737.

(18) Wang, H.; Zhao, G.; Pumera, M. Beyond Platinum: BubblePropelled Micromotors Based on $\mathrm{Ag}$ and $\mathrm{MnO}_{2}$ Catalysts. J. Am. Chem. Soc. 2014, 136, 2719-2722.

(19) Sengupta, S.; Patra, D.; Ortiz-Rivera, I.; Agrawal, A.; Shklyaev, S.; Dey, K. K.; Córdova-Figueroa, U.; Mallouk, T. E.; Sen, A. SelfPowered Enzyme Micropumps. Nat. Chem. 2014, 6, 415-422. 
(20) Wu, Z.; Li, J.; de Ávila, B. E. F.; Li, T.; Gao, W.; He, Q.; Zhang, L.; Wang, J. Water-Powered Cell-Mimicking Janus Micromotor. Adv. Funct. Mater. 2015, 25, 7497-7501.

(21) Mou, F.; Kong, L.; Chen, C.; Chen, Z.; Xu, L.; Guan, J. LightControlled Propulsion, Aggregation and Separation of Water-Fuelled $\mathrm{TiO}_{2} / \mathrm{Pt}$ Janus Submicromotors and Their "on-the-Fly" Photocatalytic Activities. Nanoscale 2016, 8, 4976-4983.

(22) Dong, R.; Zhang, Q.; Gao, W.; Pei, A.; Ren, B. Highly Efficient Light-Driven $\mathrm{TiO}_{2}-\mathrm{Au}$ Janus Micromotors. ACS Nano 2016, 10, 839-844.

(23) Jiang, H.-R.; Yoshinaga, N.; Sano, M. Active Motion of a Janus Particle by Self-Thermophoresis in a Defocused Laser Beam. Phys. Rev. Lett. 2010, 105, 268302.

(24) Xu, T.; Soto, F.; Gao, W.; Garcia-Gradilla, V.; Li, J.; Zhang, X.; Wang, J. Ultrasound-Modulated Bubble Propulsion of Chemically Powered Microengines. J. Am. Chem. Soc. 2014, 136, 8552-8555.

(25) Wang, W.; Li, S.; Mair, L.; Ahmed, S.; Huang, T. J.; Mallouk, T. E. Acoustic Propulsion of Nanorod Motors inside Living Cells. Angew. Chem., Int. Ed. 2014, 53, 3201-3204.

(26) Xu, T.; Soto, F.; Gao, W.; Dong, R.; Garcia-Gradilla, V.; Magaña, E.; Zhang, X.; Wang, J. Reversible Swarming and Separation of Self-Propelled Chemically Powered Nanomotors under Acoustic Fields. J. Am. Chem. Soc. 2015, 137, 2163-2166.

(27) Balk, A. L.; Mair, L. O.; Mathai, P. P.; Patrone, P. N.; Wang, W.; Ahmed, S.; Mallouk, T. E.; Liddle, J. A.; Stavis, S. M. Kilohertz Rotation of Nanorods Propelled by Ultrasound, Traced by Microvortex Advection of Nanoparticles. ACS Nano 2014, 8, 8300-8309.

(28) Ahmed, S.; Gentekos, D. T.; Fink, C. A.; Mallouk, T. E. SelfAssembly of Nanorod Motors into Geometrically Regular Multimers and Their Propulsion by Ultrasound. ACS Nano 2014, 8, 1105311060 .

(29) Zhang, J.; Yao, Y.; Sheng, L.; Liu, J. Self-Fueled Biomimetic Liquid Metal Mollusk. Adv. Mater. 2015, 27, 2648-2655.

(30) Tu, Y.; Peng, F.; Sui, X.; Men, Y.; White, P. B.; van Hest, J. C.; Wilson, D. A. Self-Propelled Supramolecular Nanomotors with Temperature-Responsive Speed Regulation. Nat. Chem. 2017, 9, 480-486.

(31) Wilson, D. A.; Nolte, R. J.; Van Hest, J. C. Autonomous Movement of Platinum-Loaded Stomatocytes. Nat. Chem. 2012, 4, 268-274.

(32) Alarcón-Correa, M.; Lee, T. C.; Fischer, P. Dynamic Inclusion Complexes of Metal Nanoparticles inside Nanocups. Angew. Chem. 2015, 127, 6834-6838.

(33) Peng, F.; Tu, Y.; Wilson, D. A. Micro/Nanomotors towards In Vivo Application: Cell, Tissue and Biofluid. Chem. Soc. Rev. 2017, 46, $5289-5310$

(34) Wang, H.; Moo, J. G. S.; Pumera, M. From Nanomotors to Micromotors: The Influence of the Size of an Autonomous BubblePropelled Device upon Its Motion. ACS Nano 2016, 10, 5041-5050.

(35) Gao, W.; Uygun, A.; Wang, J. Hydrogen-Bubble-Propelled Zinc-Based Microrockets in Strongly Acidic Media. J. Am. Chem. Soc. 2012, 134, 897-900.

(36) Santiago, I. Nanoscale Active Matter Matters: Challenges and Opportunities for Self-Propelled Nanomotors. Nano Today 2018, 19, $11-15$.

(37) Helden, L.; Eichhorn, R.; Bechinger, C. Direct Measurement of Thermophoretic Forces. Soft Matter 2015, 11, 2379-2386.

(38) Balasubramanian, S.; Kagan, D.; Manesh, K. M.; Calvo-Marzal, P.; Flechsig, G. U.; Wang, J. Thermal Modulation of Nanomotor Movement. Small 2009, 5, 1569-1574.

(39) Sánchez, S.; Soler, L.; Katuri, J. Chemically Powered Microand Nanomotors. Angew. Chem., Int. Ed. 2015, 54, 1414-1444.

(40) Gorostiza, P.; Isacoff, E. Y. Optical Switches for Remote and Noninvasive Control of Cell Signaling. Science 2008, 322, 395-399.

(41) Xu, L.; Mou, F.; Gong, H.; Luo, M.; Guan, J. Light-Driven Micro/Nanomotors: From Fundamentals to Applications. Chem. Soc. Rev. 2017, 46, 6905-6926.
(42) Xuan, M.; Mestre, R.; Gao, C.; Zhou, C.; He, Q.; Sánchez, S. Noncontinuous Super-Diffusive Dynamics of a Light-Activated Nanobottle Motor. Angew. Chem., Int. Ed. 2018, 57, 6838-6842.

(43) Ragazzon, G.; Baroncini, M.; Silvi, S.; Venturi, M.; Credi, A. Light-Powered Autonomous and Directional Molecular Motion of a Dissipative Self-Assembling System. Nat. Nanotechnol. 2015, 10, 7075

(44) Erbas-Cakmak, S.; Leigh, D. A.; McTernan, C. T.; Nussbaumer, A. L. Artificial Molecular Machines. Chem. Rev. 2015, 115, 1008110206.

(45) Baigl, D. Photo-Actuation of Liquids for Light-Driven Microfluidics: State of the Art and Perspectives. Lab Chip 2012, 12, 3637-3653.

(46) Uchida, E.; Azumi, R.; Norikane, Y. Light-Induced Crawling of Crystals on a Glass Surface. Nat. Commun. 2015, 6, 7310.

(47) Solovev, A. A.; Smith, E. J.; BofBufon, C. C.; Sanchez, S.; Schmidt, O. G. Light-Controlled Propulsion of Catalytic Microengines. Angew. Chem., Int. Ed. 2011, 50, 10875-10878.

(48) Chen, C.; Mou, F.; Xu, L.; Wang, S.; Guan, J.; Feng, Z.; Wang, Q.; Kong, L.; Li, W.; Wang, J.; Zhang, Q. Light-Steered Isotropic Semiconductor Micromotors. Adv. Mater. 2017, 29, 1603374.

(49) Loh, I. Y.; Cheng, J.; Tee, S. R.; Efremov, A.; Wang, Z. From Bistate Molecular Switches to Self-Directed Track-Walking Nanomotors. ACS Nano 2014, 8, 10293-10304.

(50) Yang, Y.; Goetzfried, M. A.; Hidaka, K.; You, M.; Tan, W.; Sugiyama, H.; Endo, M. Direct Visualization of Walking Motions of Photocontrolled Nanomachine on the DNA Nanostructure. Nano Lett. 2015, 15, 6672-6676.

(51) Liu, M.; Hou, R.; Cheng, J.; Loh, I. Y.; Sreelatha, S.; Tey, J. N.; Wei, J.; Wang, Z. Autonomous Synergic Control of Nanomotors. ACS Nano 2014, 8, 1792-1803.

(52) Eskandarloo, H.; Kierulf, A.; Abbaspourrad, A. Light-Harvesting Synthetic Nano- and Micromotors: A Review. Nanoscale 2017, 9, $12218-12230$.

(53) Shao, L.; Yang, Z.-J.; Andren, D.; Johansson, P.; Kall, M. Gold Nanorod Rotary Motors Driven by Resonant Light Scattering. ACS Nano 2015, 9, 12542-12551.

(54) Alkanawati, M. S.; Wurm, F. R.; Thérien-Aubin, H.; Landfester, K. Large-Scale Preparation of Polymer Nanocarriers by High-Pressure Microfluidization. Macromol. Mater. Eng. 2018, 303, 1700505.

(55) Tang, S. Y.; Shridharan, P.; Sivakumar, M. Impact of Process Parameters in the Generation of Novel Aspirin Nanoemulsions Comparative Studies between Ultrasound Cavitation and Microfluidizer. Ultrason. Sonochem. 2013, 20, 485-497.

(56) Philipse, A. P.; Van Bruggen, M. P.; Pathmamanoharan, C. Magnetic Silica Dispersions: Preparation and Stability of SurfaceModified Silica Particles with a Magnetic Core. Langmuir 1994, 10, 92-99.

(57) Wibowo, D.; Hui, Y.; Middelberg, A. P. J.; Zhao, C.-X. Interfacial Engineering for Silica Nanocapsules. Adv. Colloid Interface Sci. 2016, 236, 83-100.

(58) Landfester, K. Synthesis of Colloidal Particles in Miniemulsions. Annu. Rev. Mater. Res. 2006, 36, 231-279.

(59) Hyuk Im, S.; Jeong, U.; Xia, Y. Polymer Hollow Particles with Controllable Holes in Their Surfaces. Nat. Mater. 2005, 4, 671-675.

(60) Chen, C.; Wang, H.; Han, C.; Deng, J.; Wang, J.; Li, M.; Tang, M.; Jin, H.; Wang, Y. Asymmetric Flasklike Hollow Carbonaceous Nanoparticles Fabricated by the Synergistic Interaction between Soft Template and Biomass. J. Am. Chem. Soc. 2017, 139, 2657-2663.

(61) Yamashina, M.; Sei, Y.; Akita, M.; Yoshizawa, M. Safe Storage of Radical Initiators within a Polyaromatic Nanocapsule. Nat. Commun. 2014, 5, 4662.

(62) Dunderdale, G.; Ebbens, S.; Fairclough, P.; Howse, J. Importance of Particle Tracking and Calculating the Mean-Squared Displacement in Distinguishing Nanopropulsion from Other Processes. Langmuir 2012, 28, 10997-11006.

(63) Kapral, R. Perspective: Nanomotors without Moving Parts that Propel Themselves in Solution. J. Chem. Phys. 2013, 138, No. 020901. 
(64) Wang, W.; Dong, Z.; Xia, P.; Yan, D.; Zhang, Q. Reverse Atom Transfer Radical Polymerization of Methyl Acrylate Using AIBN as the Initiator under Heterogeneous Conditions. Macromol. Rapid Commun. 1998, 19, 647-649.

(65) Juang, R. S.; Liang, J. F. Thermal Decomposition of Azobisisobutyronitrile Dissolved in Xylene in the Presence of Tin (IV) Chloride. J. Chem. Technol. Biotechnol. 1992, 55, 379-383.

(66) Li, L.; Wang, J.; Li, T.; Song, W.; Zhang, G. A Unified Model of Drag Force for Bubble-Propelled Catalytic Micro/Nano-Motors with Different Geometries in Low Reynolds Number Flows. J. Appl. Phys. 2015, 117, 104308.

(67) Michelin, S.; Lauga, E. Geometric Tuning of Self-Propulsion for Janus Catalytic Particles. Sci. Rep. 2017, 7, 42264.

(68) Ke, H.; Ye, S.; Carroll, R. L.; Showalter, K. Motion Analysis of Self-Propelled Pt-Silica Particles in Hydrogen Peroxide Solutions. J. Phys. Chem. A 2010, 114, 5462-5467.

(69) Ebbens, S. J.; Howse, J. R. Direct Observation of the Direction of Motion for Spherical Catalytic Swimmers. Langmuir 2011, 27, $12293-12296$.

(70) Valadares, L. F.; Tao, Y. G.; Zacharia, N. S.; Kitaev, V.; Galembeck, F.; Kapral, R.; Ozin, G. A. Catalytic Nanomotors: SelfPropelled Sphere Dimers. Small 2010, 6, 565-572. 